Pacific Journal of Mathematics

COEFFICIENT ESTIMATES FOR CERTAIN MULTIVALEN 


\title{
COEFFICIENT ESTIMATES FOR CERTAIN MULTIVALENT FUNCTIONS
}

\author{
RONALD J. LEACH
}

\begin{abstract}
We prove the Goodman conjecture for a class of multivalent functions including close-to-convex functions under the restriction that the coefficients are real. We obtain similar results for other classes of multivalent functions.
\end{abstract}

1. Introduction. Let $S$ denote the class of all functions $f$ analytic and univalent in the unit disc $U$ with $f(0)=0$ and $f^{\prime}(0)=1$ and let $S^{*}$ and $K$ denote the subclasses of starlike and close-toconvex functions, respectively. Several authors ([3], [5], [6], [9]) have defined multivalent analogs of these subclasses. A commonly used definition is that $f \in S(p)$, the class of $p$-valent starlike functions, if and only if there are numbers $z_{j}$ with $\left|z_{j}\right|<1$ and a function $g \in S^{*}$ such that

$$
f(z)=\prod_{m=1}^{p} \uparrow\left(z, z_{m}\right) g(z)^{p},
$$

where

$$
\psi\left(z, z_{m}\right)=\left(z-z_{m}\right)\left(1-\bar{z}_{m} z\right) / z
$$

A function $g(z)$ is said to be a Bazilevic function of order $\alpha$, $\alpha>0$, if

$$
g(z)=\left[\alpha \int_{0}^{z} \sigma^{\alpha}(\xi) h(\xi) \xi^{-1} d \xi\right]^{1 / \alpha},
$$

where $\sigma$ is a univalent starlike function and $\operatorname{Re} h(z)>0, h(0)=1$.

If $g$ belongs to the class $B(p)$ of univalent Bazilevic functions of order $p$, then $f$ belongs to the class $K(p)$ of $p$-valently close-toconvex functions [6]. The representation (1.1) holds for multivalent analogs of several classes of vnivalent starlike functions [7]. A similar representation holds for the class $V_{k}(p)$ multivalent functions of bounded boundary rotation, with $f$ and $g$ replaced by their derivatives [8].

We say that a function $f$ belongs to the class $M(p)$ of multivalent functions of order $p$ if there are $z_{1}, \cdots, z_{p}$ with $\left|z_{m}\right| \leqq 1$ and a function $g \in S$ such that (1.1) holds. We note that $S(p)$ and $K(p)$ are proper subsets of $M(p)$.

This paper is divided into four sections. In $\S 2$, we prove a preliminary result on the coefficients of a polynomial in $z(1-z)^{-2}$. In $\S 3$, we obtain the Goodman conjecture for functions in $M(p)$ with 
real coefficients. Finally, in $\S 4$ we obtain as corollaries some coefficient conjectures for functions with real coefficients in $K(p), V_{k}(p)$ and certain other classes.

2. Preliminary results. The following two results are motivated by a result of Goodman [3] and Goodman and Robertson [4]. They show that the extremal functions for the coefficient problem in several classes of functions are the same.

LEMMA 1. If $f_{k}(z)=\sum_{n=p-k}^{\infty} a_{n}^{(k)} z^{n}=\sum_{n=p-k}^{p} c_{n}^{(k)} u^{n}$ where $u(z)=$ $z(1-z)^{-2}$ and $z_{k+1}$ is real, then $f_{k+1}(z)=\psi\left(z, z_{k+1}\right) f_{k}(z)=\sum_{n=p-k-1}^{\infty} a_{n}^{(k+1)} z^{n}$ is also a polynomial $\sum_{n=p-k-1}^{p} c_{n}^{(k+1)} u^{n}$ in $u$. If $\operatorname{sgn} a_{j}^{(k)}=(-1)^{p-j}$ for $p-k \leqq j \leqq p$, then for fixed $n$ and fixed $z_{k+1}>0$, the signs of $a_{j}^{(k+1)}$ alternate for $p-k-1 \leqq j \leqq p$ and for $n>p$,

$$
\left|\alpha_{n}^{(k+1)}\right|=\sum_{j=1}^{p} D(p, n, j)(-1)^{p-j} a_{j}^{(k+1)},
$$

where

$$
D(p, n, j)=\frac{2 j(n+p) !}{(p+j) !(p-j) !(n-p-1) !\left(n^{2}-j^{2}\right)} .
$$

Proof. Since $z_{k+1}$ is real,

$$
\begin{aligned}
\psi\left(z, z_{k+1}\right) & =\frac{-z_{k+1}+\left(1+\left(z_{k+1}\right)^{2}\right) z-z_{k+1} z^{2}}{z} \\
& =1+\left(z_{k+1}\right)^{2}-z_{k+1}\left(1+z^{2}\right) / z \\
& =\left(1-z_{k+1}\right)^{2}-z_{k+1} / u
\end{aligned}
$$

which proves the first statement. To prove the second statement note that

$$
\begin{aligned}
a_{n}^{(k+1)}= & -z_{k+1} a_{n+1}^{(k)}+\left(1+\left(z_{k+1}\right)^{2}\right) a_{n}^{(k)}-z_{k-1} a_{n-1}^{(k)} \\
= & -z_{k+1}(-1)^{p-n-1}\left|a_{n+1}^{(k)}\right|+\left(1+\left(z_{k+1}\right)^{2}\right)(-1)^{p-n}\left|a_{n}^{(k)}\right| \\
& -z_{k+1}(-1)^{p-n+1}\left|a_{n-1}^{(k)}\right| \\
= & (-1)^{p-n}\left[z_{k+1}\left|a_{n+1}^{(k)}\right|+\left(1+\left(z_{k+1}\right)^{2}\right)\left|a_{n}^{(k)}\right|+z_{k+1}\left|a_{n-1}^{(k)}\right|\right]
\end{aligned}
$$

where we define $a_{0}^{(k)}=a_{-1}^{(k)}=0$. The remaining statement was proved in [3]. (See also [4].)

Lemma 2. Suppose $z_{m}=\bar{z}_{m}$. Then there are real numbers $b_{0}, b_{1}$, and $b_{2}$ so that

$$
\psi\left(z, z_{m}\right) \psi\left(z, \bar{z}_{m}\right)=b_{0}+b_{1} u^{-1}+b_{2} u^{-2},
$$

where $u(z)=z(1-z)^{-2}$. 
Proof. From (1.2),

$$
\begin{aligned}
\psi\left(z, z_{m}\right) \psi\left(z, \bar{z}_{m}\right)= & \left|z_{m}\right|^{2}\left[\frac{1}{z^{2}}+z^{2}\right]+\left(1+\left|z_{m}\right|^{2}\right)\left(z_{m}+\bar{z}_{m}\right)\left(\frac{1}{z}+z\right) \\
& +\left(1+\left|z_{m}\right|^{2}\right)^{2}+z_{m}^{2}+\bar{z}_{m}^{2}
\end{aligned}
$$

and the result follows after a short calculation.

3. The Goodman conjecture for $M(p)$. In 1948, A. W. Goodman [2] conjectured that if $f(z)=\sum_{0}^{\infty} a_{n} z^{n}$ is analytic and at most $p$ valent in $|z|<1$, then for $1 \leqq j \leqq p<n$,

$$
\left|a_{n}\right| \leqq \sum_{j=1}^{p} D(p, n, j)\left|a_{j}\right|,
$$

where

$$
D(p, n, j)=\frac{2 j(n+p) !}{(p+j) !(p-j) !(n-p-1) !\left(n^{2}-j^{2}\right)} .
$$

This conjecture reduces to the Bieberbach conjecture $\left|a_{n}\right| \leqq n\left|a_{1}\right|$ if $p=1$. The conjecture has been proved by A. W. Goodman and M. S. Robertson [4] if $f \in S(p)$ and each $a_{n}$ is real and by A. E. Livingston if $f \in K(p)$ for $n=p+1$ with no restrictions on $a_{1}, \cdots, a_{p}$ [9] and if $f \in K(p)$ for all $n \geqq p+1$ provided $a_{1}=a_{2}=\cdots=a_{p-2}=0$ [10].

We will prove that (3.1) holds if $f \in M(p)$ and has all coefficients real. As a corollary we will obtain the Goodman conjecture for functions in $K(p)$ with real coefficients. Our method of proof is induction on the number $k$ of $z_{j}$ that are not at the origin.

THEOREM 1. Suppose $f(z)=\sum_{0}^{\infty} a_{n} z^{n} \in M(p)$ and that each $a_{n}$ is real. Then for $1 \leqq j \leqq p<n$,

$$
\left|a_{n}\right| \leqq \sum_{j=1}^{p} \frac{2 j(n+p) !}{(p+j) !(p-j) !(n-p-1) !\left(n^{2}-j^{2}\right)}\left|a_{j}\right| .
$$

Proof. Suppose first that

$$
f(z)=\prod_{m=1}^{p} \psi\left(z, z_{m}\right) \cdot g(z)^{p}
$$

and that each $z_{m}$ is real. We define the functions $f_{k}(z), 0 \leqq k \leqq p$, by

$$
\begin{gathered}
f_{0}(z)=(g(z))^{p} \\
f_{k+1}(z)=\psi\left(z, z_{k+1}\right) f_{k}(z) .
\end{gathered}
$$

We note that the power series expansion of $f_{k}$ is of the form

$$
f_{k}(z)=\sum_{n-p-k}^{\infty} a_{n}^{(k)} z^{k}
$$


Since each $z_{k}$ is real, $z_{k+1}=\bar{z}_{k+1}$ and (3.5) yields

$$
\begin{aligned}
f_{k+1}(z) & =\sum_{n=p-k-1}^{\infty} a_{n}^{(k+1)} z^{n} \\
& =\frac{\left(z-z_{k+1}\right)\left(1-z_{k+1} z\right)}{z} f_{k}(z) \\
& =\frac{\left(z-z_{k+1}\right)\left(1-z_{k+1} z\right)}{z} \sum_{n=p-k}^{\infty} a_{n} z^{n} .
\end{aligned}
$$

A short calculation shows

$$
a_{n}^{(k+1)}=-z_{k+1} a_{n+1}^{(k)}+\left(1+\left(z_{k+1}\right)^{2}\right) a_{n}^{(k)}-z_{k+1} a_{n-1}^{(k)}
$$

where we define $a_{p-k-1}^{(n)}=a_{p-k-2}^{(n)}=0$ to make the formula simpler.

We begin by showing that (3.3) holds for $k=0$. If $g(z)=z+$ $b_{2} z^{2}+\cdots$, then from (2.4) and (2.6),

$$
\sum_{n=p}^{\infty} a_{n}^{(0)} z^{n}=\left[z+\sum_{n=2}^{\infty} b_{n} z^{n}\right]^{p} \text {. }
$$

Since each $a_{n}$ is real, each $b_{n}$ is real and hence $\left|b_{n}\right| \leqq n$ with equality for each $n$ only if $g(z)=z(1-z)^{-2}$ or $z(1+z)^{-2}$. An elementary computation shows that

$$
\left|\alpha_{n}^{(0)}\right| \leqq D(p, n, p)\left|\alpha_{p}^{(0)}\right|
$$

which proves the coefficient conjecture for $f_{0}$.

Suppose now the conjecture is true for $f_{k}$. That is, for $n>p$,

$$
\left|a_{n}^{(k)}\right| \leqq \sum_{j=p-k}^{p} D(p, n, j)\left|a_{j}^{(k)}\right|
$$

We write (3.8) in the form

$$
a_{n}^{(k)}=\sum_{j=p-k}^{p} d(p, n, j) a_{j}^{(k)},
$$

where we choose the $d(p, n, j)$ by

$$
a_{n}^{(0)}=d(p, n, p) a_{p}^{(0)}
$$

and we choose the remaining $d(p, n, j), p-k \leqq j \leqq p-1$ so that

$$
|(p, n, j)| \leqq D(p, n, j) \text {. }
$$

The choice of $d(p, n, j)$ is clearly not unique. We will show that there is a method of choosing $d(p, n, j), p-k \leqq j \leqq p$ which leads naturally to a $d(p, n, p-k-1)$ such that

$$
a_{n}^{(k+1)}=\sum_{n=p-k-1}^{p} d(p, n, j) a_{j}^{(k+1)}
$$


where the additional expression $d(p, n, p-k-1)$ satisfies (3.10) with $j=p-k-1$. From (3.7) we obtain

$$
\begin{aligned}
a_{n}^{(k+1)}= & \sum_{j=p-k-1}^{p}\left[-z_{k+1} d(p, n+1, j)+\left(1+\left(z_{k+1}\right)^{2}\right) d(p, n, j)\right. \\
& \left.-z_{k+1} d(p, n-1, j)\right] \alpha_{j}^{(k)},
\end{aligned}
$$

where for simplicity we define $d(m, n, j)$ for $m \leqq p$ as 1 if $m=j$ and 0 if $m \neq j$. Let

$$
\begin{aligned}
a_{n}^{(k+1)}= & \sum_{j=p-k-1}^{p} c(p, n, j) a_{j}^{(k+1)} \\
= & \sum_{j=p-k-1}^{p-1} c(p, n, j)\left[-z_{k+1} a_{j+1}^{(k)}\right. \\
& \left.+\left(1+\left(z_{k+1}\right)^{2}\right) a_{j}^{(k)}-z_{k+1} a_{j-1}^{(k)}\right] \\
& +c(p, n, p)\left[-z_{k+1} \sum_{j=p-k}^{p} d(p, p+1, j) \alpha_{j}^{(k)}\right. \\
& \left.+\left(1+\left(z_{k+1}\right)^{2}\right) a_{p}^{(k)}-z_{k+1} a_{p-1}^{(k)}\right] .
\end{aligned}
$$

Equating coefficients of $\alpha_{j}$ in (3.12) and (3.13) we obtain

$$
-z_{k+1}[c(p, n, j-1)+c(p, n, j+1)+c(p, n, p) d(p, p+1, j)]
$$

$(3.14)+\left(1+\left(z_{k+1}\right)^{2}\right) c(p, n, j)$

$$
=-z_{k+1}[d(p, n+1, j)+d(p, n-1, j)]+\left(1+\left(z_{k+1}\right)^{2}\right) d(p, n, j),
$$

where we have set $c(p, n, p+1)=0$. One solution to the system (3.14) is

$$
\begin{aligned}
c(p, n, j)= & d(p, n, j) \quad p-k \leqq j \leqq p \\
c(p, n, p-k-1)= & d(p, n+1, p-k) \\
& +d(p, n-1, p-k)-d(p, n, p-k+1) \\
& -d(p, n, p) d(p, p+1, p-k)
\end{aligned}
$$

and we note that this solution is independent of $z_{k+1}$. Since $S$ is compact in the topology of uniform convergence on compact subsets of $\mid z<1$ and since the only restriction on the $z_{m}$ is that $\left|z_{m}\right| \leqq 1$, $M(p)$ is compact in the topology of uniform convergence on compact subsets of $|z|<1$. Consequently, any subset of $M(p)$ where either the $z_{m}$ are fixed or some of the coefficients have fixed absolute value is also compact in this topology.

Let $f_{k+1}=\sum_{n=p-k-1}^{\infty} a_{n} z^{n}$ be an extremal function in the set $\left\{f_{k+1}\right\}$ of all $f_{k+1}(z)$ of the form (3.5). That is, suppose there is a nonnegative constant $E=E(p, n, p-k-1)$ such that

$$
\left|a_{n}^{(k+1)}\right|=\sum_{j=p-k}^{p} D(p, n, j)\left|a_{j}^{(k+1)}\right|+E\left|a_{p-k-1}^{(k+1)}\right|
$$


and for any $f_{k+1}=\Sigma b_{n}^{(k+1)} z^{n}$,

$$
\left|b_{n}^{(k+1)}\right| \leqq \sum_{j=p-k}^{p} D(p, n, j)\left|b_{j}^{(k+1)}\right|+E\left|b_{p-k-1}^{(k+1)}\right| .
$$

Because of equation (2.1), the function $f_{k+1}$ of Lemma 1 shows that $E \geqq D(p, n, p-k-1)$. We claim that if $f_{k+1}$ is extremal for $\left\{f_{k+1}\right\}$, then the function $f_{k}$ defined by (2.5) is extremal for $\left\{f_{k}\right\}$. Suppose not. Then

$$
\left|\alpha_{n}^{(k)}\right|<\sum_{j=p-k}^{p} D(p, n, j)\left|\alpha_{j}^{(k)}\right| .
$$

Since by (3.7) $a_{m}^{(k+1)}$ is a continuous function of $z_{k+1}, m \geqq p-k-1$,

$$
\left|\alpha_{n}^{(k+1)}\right|<\sum_{j=p-k}^{p} D(p, n, j)\left|\alpha_{j}^{(k+1)}\right|+E\left|\alpha_{p-k-1}^{(k+1)}\right|
$$

at least for $z_{k+1}$ sufficiently close to 0 . Since $E \geqq D(p, n, p-k-1)$, there must be a $z_{k+1}$ for which $a_{n}^{(k+1)}$ (considered as a function of $\left.z_{k+1}\right)$ must satisfy

$$
\left|a_{n}^{(k+1)}\right|=\sum_{j=p-k-1}^{p} D(p, n, j)\left|a_{j}^{(k+1)}\right| .
$$

The set of all extremal functions $\left\{f_{k+1}\right\}$ for which $\left|a_{p-k}^{(k)}\right|, \cdots,\left|a_{p}^{(k)}\right|$ and $\left|a_{n}^{(k)}\right|$ are fixed and (3.17) holds is a normal and compact family and hence there is an $\varepsilon>0$ so that

$$
\left|\alpha_{n}^{(k+1)}\right|<\sum_{j=p-k-1}^{p} D(p, n, j)\left|\alpha_{j}^{(k+1)}\right|
$$

for $\left|z_{k+1}\right|<\varepsilon$ and (3.18) holds if $\left|z_{k+1}\right|=\varepsilon$. We may suppose $z_{k+1}<0$ since if not we consider $f(-z)$. Our extremal function then solves the problem of maximizing $\left|a_{n}^{(k+1)}\right|$ when $z_{k+1}<0$ is fixed and $\left|a_{p-k}^{(k)}\right|, \cdots,\left|a_{p}^{(k)}\right|$ are fixed and (3.17) holds.

We will need the following result.

LEMMA 3. Let $g_{k}(z)=\sum_{p-k}^{\infty} b_{n}^{(k)} z^{n}$, with $\left|b_{p-k}^{(n)}\right|, \cdots,\left|b_{p}^{(n)}\right|$ fixed and let $z_{k+1}<0$. Then $\left|b_{n}^{(k+1)}\right|$ is maximal when the signs of $b_{j}^{(n)}$ alternate for $p-k \leqq j \leqq p$.

Proof. From (3.7) and (3.9),

$$
\begin{aligned}
b_{n}^{(k+1)}= & -z_{k+1} b_{n+1}^{(k)}+\left(1+\left(z_{k+1}\right)^{2}\right) b_{n}^{(k)}-z_{k+1} b_{n-1}^{(k)} \\
= & \sum_{j=p-k}^{p}\left[-z_{k+1} d(p, n+1, j)+\left(1+\left(z_{k+1}\right)^{2}\right) d(p, n, j)\right. \\
& \left.\left.-z_{k+1} d(p, n-1, j)\right] b_{j}^{k}\right) \\
= & \sum_{j=p-k}^{p}\left\{\left[-z_{k+1} d(p, n+1, j)+\left(1+\left(z_{k+1}\right)^{2}\right) d(p, n, j)\right.\right. \\
& \left.\left.-z_{k+1} d(p, n-1, j)\right] \sum_{r=0}^{j+k-p} h(r, j) b_{j-r-1}^{(k+1)}\right\}
\end{aligned}
$$


where $h(r, j)$ is a rational function of $z_{k+1}$ obtained by solving (3.7) for $b_{j}^{(n)}$. An elementary calculation shows that

$$
\operatorname{sgn} h(r, j)=(-1)^{r} \text {. }
$$

Since $z_{k+1}<0$, the maximum value of $\left|a_{n}^{(k+1)}\right|$ must occur when

$$
\operatorname{sgn} d(p, n+1, j)=\operatorname{sgn} d(p, n, j)=\operatorname{sgn} d(p, n-1, j)
$$

and the signs must alternate because of (3.19), which proves the lemma.

By Lemma 3, for fixed $\left|a_{p-k}^{(k)}\right|, \cdots,\left|a_{p}^{(k)}\right|$ and fixed $z_{k+1}<0,\left|a_{n}^{(k+1)}\right|$ is maximal only when $\left|\alpha_{p-k-1}^{(k+1)}\right|, \cdots,\left|\alpha_{p}^{(k+1)}\right|$ are minimal,

$$
\operatorname{sgn} d(p, n, j)=\operatorname{sgn} \alpha_{j}^{(k)} \quad(p-k \leqq j \leqq p)
$$

and these signs alternate. An elementary but lengthy calculation shows that

$$
D(p, n, p-k-1)=D(p, n+1, p-k)
$$

$$
\begin{aligned}
& +D(p, n-1, p-k)+D(p, n, p-k+1) \\
& -D(p, n, p) D(p, p+1, p-k) .
\end{aligned}
$$

We note that $\left|a_{n}^{(k+1)}\right|$ is maximal when

$$
d(p, n, j)=(-1)^{p-j} D(p, n, j) .
$$

In view of (3.19) and (3.20),

$$
d(p, n, p-k-1)=(-1)^{k+1} D(p, n, p-k-1)
$$

and therefore this maximum can occur only if

$$
\left|a_{n}^{(k)}\right|=\sum_{j=p-k}^{p} D(p, n, j)\left|a_{j}^{(k)}\right|,
$$

which contradicts (3.17). Therefore $f_{k}$ is extremal for $\left\{f_{k}\right\}$. Applying this argument to $f_{k-1}, f_{k-2}, \cdots, f_{0}$, we see that the only possible extremal functions occur when $f_{0}$ is an extremal function; that is, when $g(z)=z(1-z)^{-2}$ or $g(z)=z(1+z)^{-2}$. We may assume $g(z)=$ $z(1-z)^{-2}$ since if not we could consider $f(-z)$.

By Lemma 1, each function $f_{k}$ can be written

$$
\begin{aligned}
f_{k+1}(z) & =\sum_{n=p-k-1}^{\infty} a_{n}^{(k+1)} z^{n} \\
& =\sum_{n=p-k-1}^{p} c_{n}^{(k+1)} u^{n},
\end{aligned}
$$

where $u(z)=z(1-z)^{-2}$. By Lemma $3, a_{n}^{(k+1)}$ is maximized only when the sign of $a_{j}^{(k+1)}$ alternates for $p-k-1<j<p$, which is equiva- 
lent to the alternating of $\operatorname{sgn} a_{\rho}^{(k)}$ by Lemma 1 . It follows then from Lemma 1 that for $n>p$,

$$
\left|a_{n}^{(k+1)}\right| \leqq \sum_{j=p-k-1}^{p} D(p, n, j)\left|a_{j}^{(k+1)}\right|
$$

The result now follows if $f$ has only real zeros.

If $f$ has a nonreal zero $z_{m}$, since $f$ has real coefficients, $f$ must also have $\bar{z}_{m}$ as a zero. If we use Lemma 2 in place of Lemma 1 , the argument in this case is similar to that in the case of real zeros. We omit the straightforward modification. This completes the proof of the theorem.

4. Applications. In the proof of Theorem 1 we showed that the only possible extremal functions were of the form

$$
f(z)=\Sigma a_{n} z^{n}=\prod_{m=1}^{p} \psi\left(z, z_{m}\right) \cdot g(z)^{p},
$$

where the signs of $a_{j}$ alternate for $1 \leqq j \leqq p$ and where $g$ is an extremal function in $S$ or of the form $f(-z)$. Since functions $f$ in $K(p)$ have the representation

$$
f^{\prime}(z)=\prod_{m=1}^{p-1} \psi\left(z, z_{m}\right) g^{\prime}(z)
$$

where $g(z)=b_{p} z^{p}+\cdots$ is in $K(p)$ and $\left|b_{n}\right| \leqq D(p, n, p)\left|b_{p}\right|$ [9] with equality if and only if $g(z)=b_{p}\left[z /(1-x z)^{2}\right]^{p}$, where $x$ is a constant with $|x|=1$, the method of Theorem 1 proves

THEOREM 2. Let $f(z)=\Sigma a_{n} z^{n} \in K(p)$ and suppose each $a_{n}$ is real. Then for $n \geqq p$,

$$
\left|a_{n}\right| \leqq \sum_{j=1}^{p} D(p, n, j)\left|a_{j}\right|,
$$

where the $D(p, n, j)$ are given by (3.2).

A similar result holds for the class $V_{k}(p)$ of multivalent functions of bounded boundary rotation. Recall that $f \in V_{k}(p)$ if there is a $\rho, 0<\rho<1$ so that if $0<\rho<r<1$,

$$
\int_{0}^{2 \pi} \operatorname{Re}\left\{1+\frac{r e^{i \theta} f^{\prime \prime}\left(r e^{i \theta}\right)}{f^{\prime}\left(r e^{i \theta}\right)}\right\} d \theta=2 p \pi
$$

and

$$
\limsup _{r \rightarrow 1} \int_{0}^{2 \pi}\left|\operatorname{Re}\left\{1+\frac{r e^{i \theta} f^{\prime \prime}\left(r e^{i \theta}\right)}{f^{\prime}\left(r e^{i \theta}\right)}\right\}\right| \leqq p k \pi .
$$

It was shown in [8] that if $f \in V_{k}(p)$, then 


$$
f^{\prime}(z)=A \prod_{m-1}^{p-g} \psi\left(z, z_{m}\right) \cdot z^{p-1}\left[g^{\prime}(z)\right]^{p}
$$

where $g(z)=z+b_{2} z^{2}+\cdots, \varepsilon V_{k}(1)$. In [1], D. Brannan, J. Clunie, and W. Kirwan proved that if $g \in V_{k}(1)$, then

$$
\left|b_{n}\right| \leqq B_{n}
$$

where

$$
G(z)=\frac{1}{k}\left[\left(\frac{1+z}{1-z}\right)^{k / 2}-1\right]=\Sigma B_{n} z^{n},
$$

with equality only for $g(z)=G(z)$ or its rotations. Consequently, the technique of Theorem 1 applies and we obtain the following.

THEOREM 3. Let $f(z)=\Sigma a_{n} z^{n} \in V_{k}(p)$ and suppose each $a_{n}$ is real. Then for $n>p$,

$$
\left|a_{n}\right| \leqq \sum_{j=1}^{p} c(p, n, j)\left|a_{j}\right|
$$

where $c(p, n, j)$ is defined by

$$
\begin{aligned}
c(p, n, j) & =0 \quad(j>p), \\
c(n, p) & =\frac{1}{n} \times\left\{\text { coefficient of } z^{n-p} \text { in }\left(\sum_{1}^{\infty} m B_{m} z^{m-1}\right)^{p}\right\}
\end{aligned}
$$

and

$$
\begin{aligned}
c(p, n, p-k-1)= & c(p, n+1, p-k) \\
& +c(p, n-1, p-k)+c(p, n, p-k+1) \\
& -c(p, n, p) c(n, p+1, p-k) \\
& (0 \leqq k \leqq p-1) .
\end{aligned}
$$

This extends a result in [8] for the case $n=p+1$ if $a_{1}=\cdots=$ $a_{p-2}=0$.

We note that this technique works for any class of functions having a representation similar to (1.1) and where the coefficients of $[g(z)]^{p}$ are simultaneously maximized by a single function with real positive coefficients. This is the case for the class $S_{1}(p, \alpha)$ defined in [7].

\section{REFERENCES}

1. D. A. Brannan, J. G. Clunie, and W. E. Kirwan, On the coefficient problem for functions of bounded boundary rotation, Ann. Acad. Sci. Fenn. Ser. A.I. No. 523 (1973), $18 \mathrm{pp}$.

2. A. W. Goodman, On some determinants related to p-valent functions, Trans. Amer. Math. Soc., 63 (1948), 175-192.

3. - On the Schwarz-Christoffel transformation and p-valent functions, Trans. 
Amer. Math. Soc., 68 (1950), 204-223.

4. A. W. Goodman and M. S. Robertson, A class of multivalent functions, Trans. Amer. Math. Soc., 70 (1951), 127-136.

5. J. A. Hummel, Multivalent starlike functions, J Analyse Math., 18 (1967),133-160.

6. R. J. Leach, Multivalent Bazilevic functions, Rev. Roumaine de Math. Pures et Appl., 21 (1976), 523-527.

7. On some classes of multivalent starlike functions, Trans. Amer. Math. Soc., 209 (1975), 267-273.

8. Multivalent and meromorphic functions of bounded boundary rotation, Canad. J. Math., 27 (1975), 186-199.

9. A. E. Livingston, p-valent close-to-convex functions, Trans. Amer. Math. Soc., 115 (1965), 161-179.

10. The coefficients of multivalent close-to-convex functions, Proc. Amer. Math. Soc., 21 (1969), 545-552.

Received April 20, 1977. Research supported by NSF Grant 76-08572.

HOWARD UNIVERSITY

WASHINGTON, D.C. 20059 


\section{PACIFIC JOURNAL OF MATHEMATICS}

\section{EDITORS}

RICHARD ARENS (Managing Editor)

University of California

Los Angeles, California 90024

C. W. Curtis

University of Oregon

Eugene, OR 97403

C. C. MOORE

University of California

Berkeley, CA 94720

\section{J. DUGUNDJI}

Department of Mathematics University of Southern California Los Angeles, California 90007

R. Finn AND J. Milgram Stanford University Stanford, California 94305

ASSOCIATE EDITORS
E. F. BECKENBACH

B. H. NeUMANN

F. WOLF

K. YoshidA

\section{SUPPORTING INSTITUTIONS}

UNIVERSITY OF BRITISH COLUMBIA UNIVERSITY OF SOUTHERN CALIFORNIA CALIFORNIA INSTITUTE OF TECHNOLOGY STANFORD UNIVERSITY UNIVERSITY OF CALIFORNIA MONTANA STATE UNIVERSITY UNIVERSITY OF TOKYO UNIVERSITY OF NEVADA, RENO UNIVERSITY OF UTAH NEW MEXICO STATE UNIVERSITY OREGON STATE UNIVERSITY UNIVERSITY OF OREGON

WASHINGTON STATE UNIVERSITY UNIVERSITY OF WASHINGTON OSAKA UNIVERSITY 


\section{Pacific Journal of Mathematics}

\section{Vol. 74, No. $1 \quad$ May, 1978}

Gerald Arthur Anderson, Computation of the surgery obstruction groups

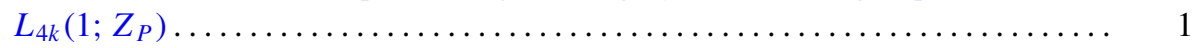

R. K. Beatson, The degree of monotone approximation ................ 5

Sterling K. Berberian, The character space of the algebra of regulated functions . . . 15

Douglas Michael Campbell and Jack Wayne Lamoreaux, Continua in the plane with

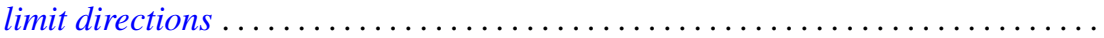

R. J. Duffin, Algorithms for localizing roots of a polynomial and the Pisot

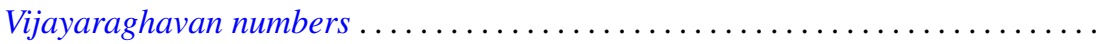

Alessandro Figà-Talamanca and Massimo A. Picardello, Functions that operate on

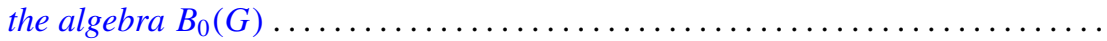

John Erik Fornaess, Biholomorphic mappings between weakly pseudoconvex

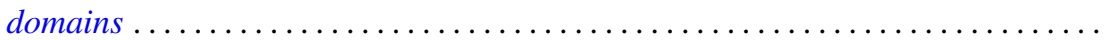

Andrzej Granas, Ronald Bernard Guenther and John Walter Lee, On a theorem of S.

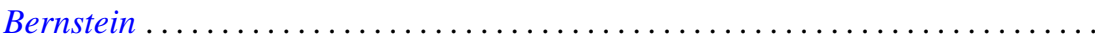

Jerry Grossman, On groups with specified lower central series quotients . .........

William H. Julian, Ray Mines, III and Fred Richman, Algebraic numbers, a constructive development . . . . . . . . . . . . . . . . . . . . . . .

Surjit Singh Khurana, A note on Radon-Nikodým theorem for finitely additive

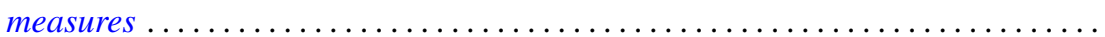

Garo K. Kiremidjian, A Nash-Moser-type implicit function theorem and nonlinear

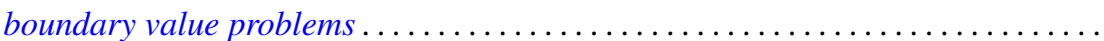

Ronald Jacob Leach, Coefficient estimates for certain multivalent functions ....

John Alan MacBain, Local and global bifurcation from normal eigenvalues. II . . 133

James A. MacDougall and Lowell G. Sweet, Three dimensional homogeneous algebras...

John Rowlay Martin, Fixed point sets of Peano continua ......

R. Daniel Mauldin, The boundedness of the Cantor-Bendixson order of some analytic sets...

Richard C. Metzler, Uniqueness of extensions of positive linear functions ..

Rodney V. Nillsen, Moment sequences obtained from restricted powers . .

Keiji Nishioka, Transcendental constants over the coefficient fields in differential elliptic function fields...

Gabriel Michael Miller Obi, An algebraic closed graph theorem

Richard Cranston Randell, Quotients of complete intersections by $\mathbf{C}^{*}$ actions . . 221

Bruce Reznick, Banach spaces which satisfy linear identities . .

Bennett Setzer, Elliptic curves over complex quadratic fields...

Arne Stray, A scheme for approximating bounded analytic functions on certain subsets of the unit disc.

Nicholas Th. Varopoulos, A remark on functions of bounded mean oscillation and bounded harmonic functions. Addendum to: "BMO functions and the

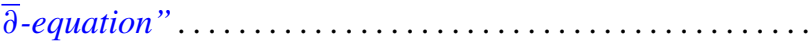

Charles Irvin Vinsonhaler, Torsion free abelian groups quasi-projective over their

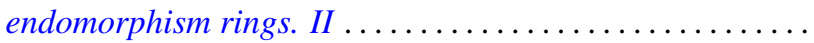

Thomas R. Wolf, Characters of $p^{\prime}$-degree in solvable groups ... 